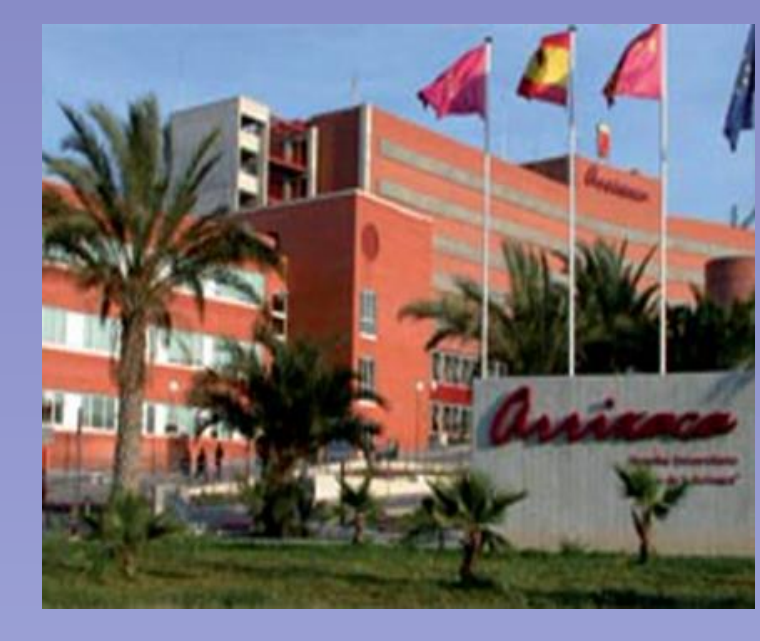

\title{
ENDOVASCULAR THERAPY (ET) EXPERIENCE IN WAKE-UP STROKES IN A REFERENCE HOSPITAL. COMPARISON WITH ENDOVASCULAR THERAPY IN KNOWN-ONSET STROKES.
}

AUTHORS:Vázquez Lorenzo, Julián Albert Lacal, Laura; Alba Isasi, María Teresa'; Parrilla Reverter, Guillermo²; Díaz Pérez, José2; Valero López, Gabriel1; Baidez Guerrero, Ana Esther1; Cabrera Maqueda, José María1; Fuentes Rumí, Luna1; García Molina, Estefanía'; Morales Ortiz, Ana1.

1.Servicio de Neurología Hospital Universitario Virgen de la Arrixaca. Murcia, España.

2.Servicio de Neurointervencionismo Hospital Universitario Virgen de la Arrixaca. Murcia, España.

\section{BACKGROUND AND PURPOSE}

Wake-up strokes can be treated with ET by selecting the patients regarding to favorable radiologic findings (ASPECTS $>6$, good colateral arteries and favorable missmatch). Checking clinical results is important to find out if selection of patients is accurate.

\section{METHODS}

We implemented a descriptive analysis in the wake-upstroke group that received ET in the period 2010-2018 in Virgen de la Arrixaca hospital. Statistical comparison with known-onset strokes that received ET was made using $\mathrm{X} 2$ and t-Student testing. SPPS 22,0 stadistics was used.

\section{RESULTS}

- Since 2010, 701 ET were performed in our Hospital. 79 of those patients suffered wake-up strokes (11.26\%).

- The medium age was 64 years-old. 51.9\% were males, 48.1 women. Cardiovascular risk factors (CVRF) found were: arterial hypertension (66.2\%), dyslipemia (40.3\%), diabetes mellitus (29.5\%), atrial fibrillation (31.2\%) and smoking habit (19.5\%). Previous to stroke, $28.3 \%$ of patients took antiaggregants and $13 \%$ anticoagulants. Cerebral media artery was the most affected(48.1\%). $10.4 \%$ had symptomatic intracranial hemorrhage. Mortality rate was $17.7 \%$. 92.2\% achieved good recanalization: Thrombolysis in Cerebral Infarction(TICI) $2 b / 3$. Rankin scale $(m R s)<2$ points three months after stroke was found in $35.1 \%$.

- Comparing with the known-onset group, we did not find significant statistical differences in CVRF. Neither we found differences in mortality rate, symptomatic hemorrhage, mRs 3 months after stroke, or TICl scales. Stenting the carotid arteries during ET was more frecuent in wake-up strokes $(p<0.05$, OR 2.2)

\begin{tabular}{|c|l|r|r|r|r|}
\hline \multicolumn{2}{|c|}{ WAKE UP-STROKE } & N & Media & $\begin{array}{c}\text { Standard } \\
\text { deviation }\end{array}$ & P value \\
\hline \multirow{2}{*}{ NIHSS $24 \boldsymbol{h}$} & No & 522 & 11,36 & 9,321 & 0,361 \\
& Yes & 84 & 11,73 & 9,279 & \\
\hline $\begin{array}{c}\text { NIHSSS } \\
\text { HOSPITAL } \\
\text { DISCHARGE }\end{array}$ & Yes & 486 & 7,81 & 7,637 & 3,322 \\
\hline NUMBER OF & No & 77 & 9,57 & 8,388 & \\
ATTEMPTS & Yes & 119 & 2,471 & 1,8078 & 2,704 \\
\hline PRODEDURE & No & 30 & 2,100 & 1,2690 & \\
LENGHT & Yes & 550 & 66,149 & 47,5085 & 0,507 \\
\hline \multirow{2}{*}{ INR } & No & 82 & 67,244 & 51,5717 & \\
& Yes & 43 & 1,3719 &, 52118 & 2,894 \\
\hline \multirow{2}{*}{ SYSTOLIC } & No & 10 & 1,1260 &, 34818 & \\
BP & Yes & 512 & 146,523 & 27,1752 & 0,55 \\
\hline \multirow{2}{*}{ DIASTOLIC } & No & 82 & 148,268 & 27,6142 & \\
BP & Yes & 512 & 77,549 & 15,1179 & 0,1 \\
\hline \multirow{2}{*}{ GLYCEMIA } & No & 82 & 78,024 & 14,5347 & \\
& Yes & 567 & 143,261 & 56,8989 & 5,399 \\
\hline \multirow{2}{*}{ AGE } & No & 91 & 142,473 & 41,4574 & \\
& Yes & 605 & 68,491 & 13,0913 & 0,477 \\
\hline
\end{tabular}

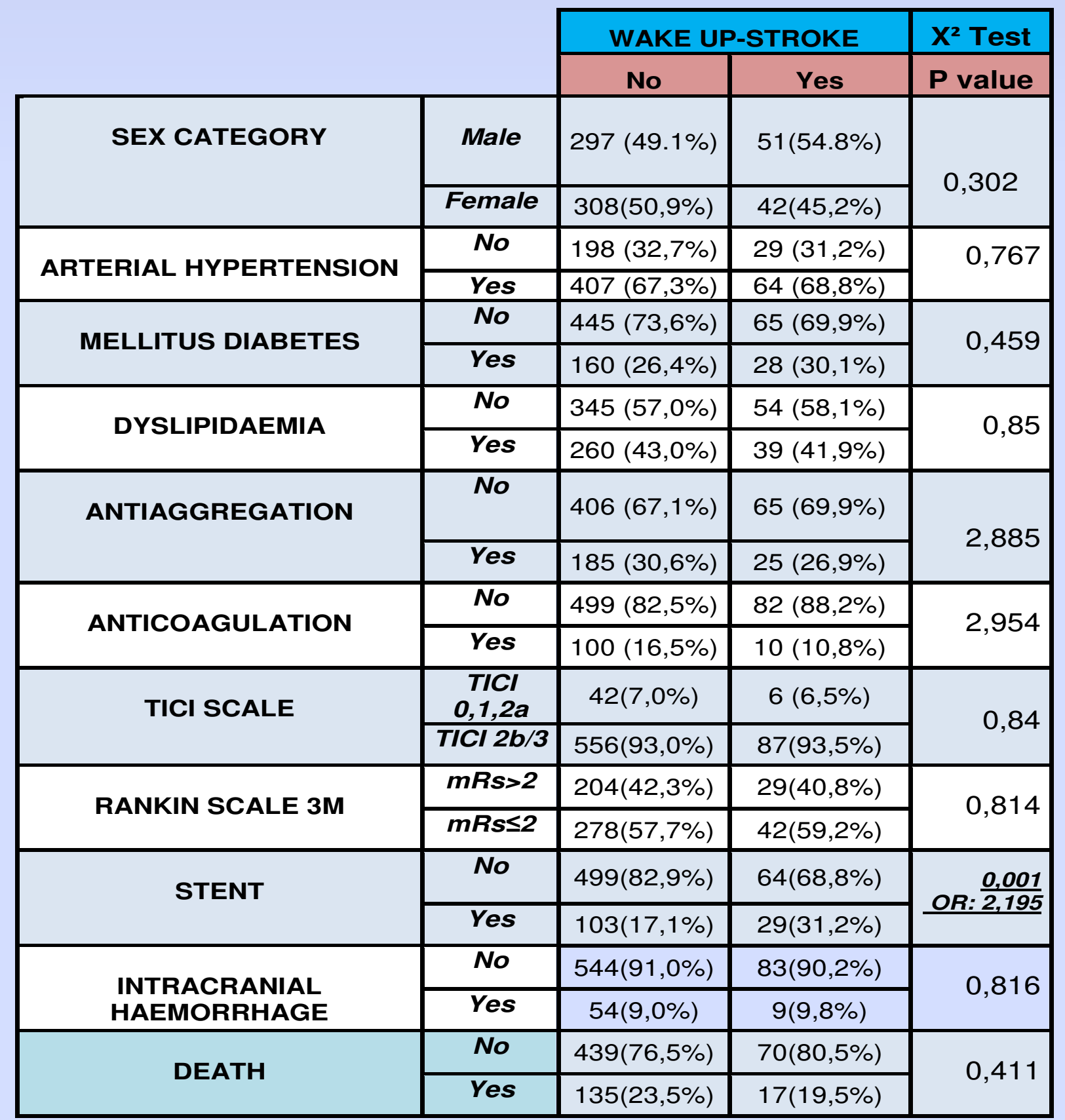

\section{CONCLUSIONS}

Endovascular treatment in wake-up strokes is as effective as in known-onset strokes if patients are selected properly. We do not found clinical or outcome differences in our sample. 